\title{
Design of a Substation Grounding System for Fast Front Overvoltage
}

\author{
L. Kevin Yuan Jet, N., N. Mohamad Nor
}

\begin{abstract}
Grounding studies allow an optimized design of grounding system to provide protection for electrical components and personnel at substation. The design of grounding system needs to comply with IEEE Standard 80:2013. Field work is conducted with Fall-of-Potential method and Wenner's method to perform measurement soil test. Data from measurement test is used to develop two-layer soil model by using Sunde's graphical method. Calculation for tolerable step voltage and touch voltage are under $50 \mathrm{~kg}$ body weight condition. Based on the findings, calculated values for tolerable step voltage and touch voltage are higher than calculated values for mesh voltage and touch voltage. The percentage of error is $7 \%$ when computed values and calculated values for tolerable voltages are compared. Higher tolerable voltages minimise the risk of getting electrical shock to any individual presents at substation during fault or lightning strike occurs. However, there is limitation in this paper related to software licensing where full simulation with grounding software required full access.
\end{abstract}

Index Terms: Grounding system, two-layer soil model, step voltage, touch voltage

Nomenclature

soil resistivity in $\Omega-\mathrm{m}$

grid area in $\mathrm{m}$

distance between electrode and potential

probe in $\mathrm{m}$

$R \quad$ soil resistance in $\Omega$

$a \quad$ spacing between two probes in $\mathrm{m}$

$K$ reflection factor

$\rho_{l} \quad$ top layer of soil resistivity in $\Omega-\mathrm{m}$

$\rho_{2} \quad$ bottom layer of soil resistivity in $\Omega-\mathrm{m}$

$\rho_{a}$ apparent resistivity of soil in $\Omega-\mathrm{m}$

$h$ depth of top layer soil in $\mathrm{m}$

$d$ diameter of ground conductor in $\mathrm{m}$

$I_{f}$ fault current in $\mathrm{kA}$

$E_{\text {step,50 }}$ tolerable step voltage for $50 \mathrm{~kg}$ body weight in $\mathrm{V}$

$E_{\text {touch,50 }}$ tolerable touch voltage for $50 \mathrm{~kg}$ body weight in $\mathrm{V}$

$I_{B} \quad$ allowable body current in A

$I_{s} \quad$ duration of shock in $\mathrm{s}$

$R_{B} \quad$ resistance of a human body in $\Omega$

$\rho_{s} \quad$ surface material resistivity in $\Omega-\mathrm{m}$

$C_{s}$ surface layer derating factor

$h_{s} \quad$ thickness of surface material in $\mathrm{m}$

Revised Manuscript Received on August 18, 2019.

L. Kevin Yuan Jet, Faculty of Engineering, Multimedia University, Cyberjaya, Malaysia.

N. Eng Eng, Faculty of Engineering, Multimedia University, Cyberjaya, Malaysia.

N. Mohamad Nor, Faculty of Engineering, Multimedia University, Cyberjaya, Malaysia.
$D \quad$ spacing between parallel conductors in $\mathrm{m}$

$n$ geometric factor of grid

$L_{T} \quad$ total length of conductor in horizontal in $\mathrm{m}$

$L_{x} \quad$ total length of grid at $\mathrm{x}$-axis in $\mathrm{m}$

$L_{y} \quad$ total length of grid at $y$-axis in $\mathrm{m}$

$L_{X} \quad$ max. length of grid conductor at $\mathrm{x}$-axis in $\mathrm{m}$

$L_{c} \quad$ total length of grid conductor in $\mathrm{m}$

$L_{R} \quad$ total length of ground rods in $\mathrm{m}$

$n_{\text {side }}$ number of grid wire pattern in one side

$L_{r} \quad$ length of ground rod at grid mesh in $\mathrm{m}$

$R_{g}$ grid resistance in $\Omega$

$I_{G} \quad$ max. grid current in $\mathrm{m}$

$S_{f}$ fault current division factor

$I_{g}$ root-mean-square symmetrical grid current in A

$D_{f} \quad$ decrement factor for entire duration of fault in $\mathrm{s}$

GPR ground potential rise in $\mathrm{kV}$

$E_{m}$ mesh voltage in $\mathrm{V}$

$E_{s} \quad$ step voltage in $\mathrm{V}$

$K_{i} \quad$ irregularity factor or correction factor for grid geometry for the simplified method

$K_{i i} \quad$ corrective weighting factor that adjusts for the effects of inner conductors on the corner mesh for simplified method

\section{INTRODUCTION}

Since high magnitude of voltage always involved in power transmission, all substations need to have grounding system for protection purpose. Grounding system is built beneath the soil where substation is located. The grid itself consists of a number of ground rods and conductors with the arrangement either in square, rectangular or irregular form depending on the layout of the substation. When lightning strikes or fault from any electrical components happen at the substation, the grounding system acts as protection system. It provides a pathway for these currents flow directly into earth instead to other electrical components or personnel at that time.

Reference mentions that Wenner's method or known as four-point method is required to perform soil measurement test [1]. Data from the tests are interpreted for soil resistivity based on type of soil model [2]. Uniform soil model and two-layer soil model are commonly used model in grid design. Reference states that two-layer soil model is a good approximation for analysis purpose [3]. Soil modelling is done by either inspection of resistivity measurement or computer algorithm. Sunde's graphical method is the first method in soil modelling and it still be applicable for two-layer soil model without

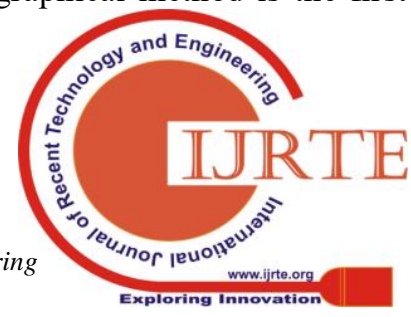




\section{Design of A Substation Grounding System for Fast Front Overvoltage}

computer algorithm as mentioned in reference [4]. Simulation of grid design with computer software such as CDEGS (Current Distribution Electromagnetic Fields, Grounding and Soil Structure Analysis), ETAP (Electrical Transient Analysis Power) and Matlab are used to verify measured data in some papers [5][6][7]. Grounding system is relatively an interesting topic especially when comes to design part. This is because many considerations that need to be taken based on par IEEE Std 80;2013 requirement [8]. In this paper, there are three main objectives; to design grounding system for a medium-sized substation, to evaluate the safety criteria for step and touch voltages via grid design as well as to compare the computed values and calculated values for tolerable voltages.

\section{METHODOLOGY}

\section{A. Grid design flow chart}

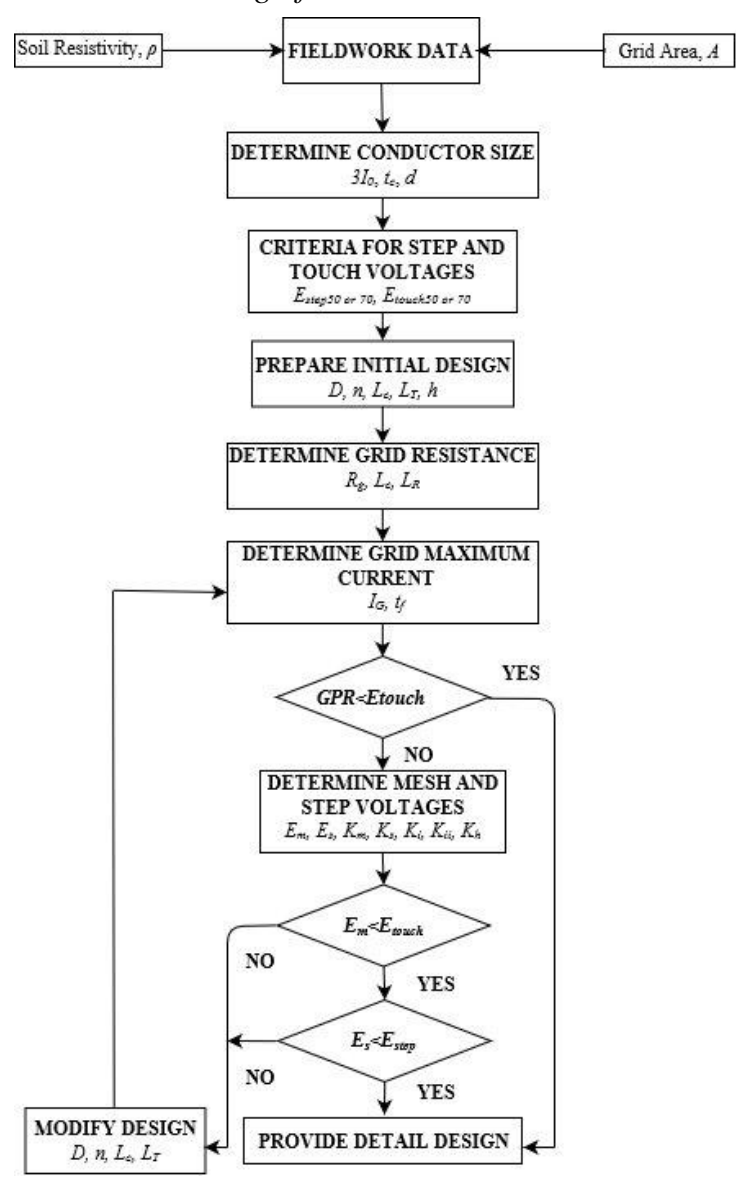

Fig. 1. The flow chart of grid design [8].

\section{B. Safety Criteria of Step and Touch Voltages}

Factors that could contribute to electric shock accidents are the presence of fault current with large magnitude, location of grounding system, soil resistivity, effect of current density on earth surface, insufficient contact resistance to limit current flow through the body of any individual who present during fault and improper duration of fault to recover. All the factors are linked with step voltage and touch voltage which determined the safety criteria of a grounding system. The safety criteria of the grounding system are achieved if tolerable step voltage is higher than the step voltage and tolerable touch voltage is higher than the mesh voltage. To strengthen more in safety criteria of the grounding system, it was recommended that a thin layer of surface materials required. With the presence of surface material, these materials were usually high resistivity spread on the top layer of soil above the ground grid to increase the contact resistance between soil and feet of persons in substation.

\section{Fieldwork Data}

\section{1) Four-point method}

Four-point method was used to measure the soil resistivity of the particular area. Obtaining the soil resistivity was vital with this method as it gave accurate value. It was usually done with the set-up arrangement of four probes where there was specific spacing between them as shown in Fig. 2. The term of $a$ represented the spacing between two probes whereas the term of $b$ represented the burial depth of probe. Then all of the probes and electrode were connected to the earth tester, Megger. The set-up arrangement was conducted on 24 January 2018 at the vicinity area of incubator building. During the measurement day took place, the condition of soil was wet and mostly contained high moisture.

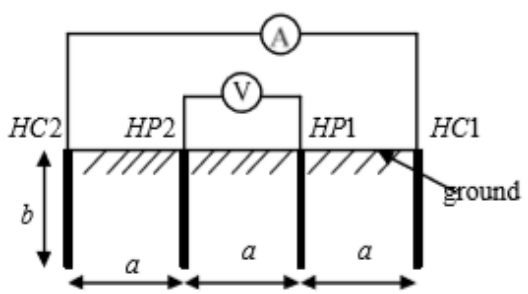

Fig. 2. The set-up arrangement for four-point method.

\section{2) Sunde's graphical method}

Sunde's graphical method is an alternate way to develop approximate two-layer soil model besides computer modelling. This method was also used to obtain the depth of layer of soil, $h$. As shown in Fig. 3, the method can determine the parameters $\rho_{1}$ and $\rho_{2}$ together with the plotted graph.

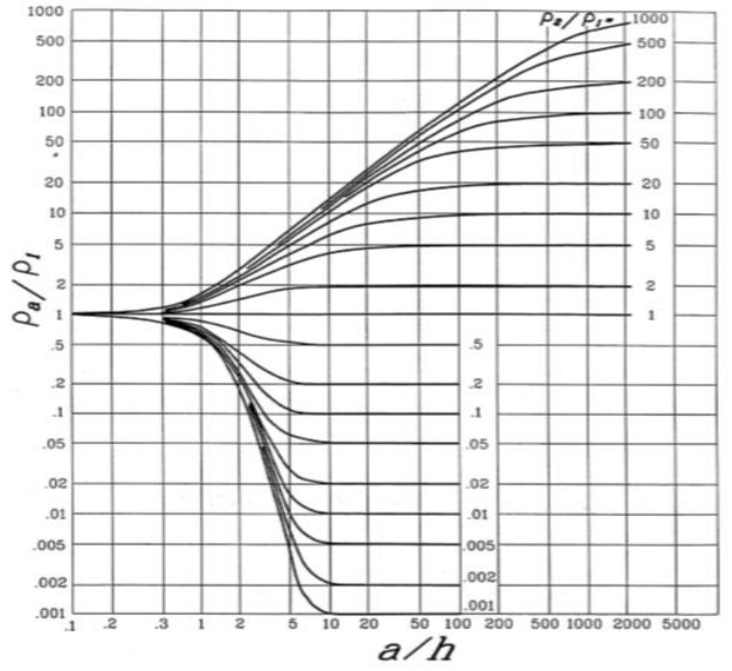

Fig. 3. The curves of Sunde's graphical method. 
The following were the procedures of using Sunde's graphical method;

a) A graph of apparent resistivity, $\rho_{a}$ versus spacing between the electrodes was plotted.

b) $\rho_{1}$ and $\rho_{2}$ were estimated from the plotted graph. $\rho_{1}$ was obtained in which $\rho_{a}$ corresponding to a smaller spacing whereas $\rho_{2}$ was obtained from a larger spacing. In case the field data were not enough, the plotted graph was extended allowing these values can be obtained.

c) $\rho_{2} / \rho_{1}$ was determined and a curve on the Sunde graph in Fig. 3 was selected. The selected curve was either matched closely or interpolated where a new curve would be drawn on the graph.

d) Within the region of the $\rho_{2} / \rho_{I}$ curve of Sunde graph, the value on the y-axis was selected.

e) One the x-axis, the corresponding value of $a / h$ was obtained.

f) Then the selected value of $\rho_{a} / \rho_{1}$ was multiplied with $\rho_{l}$ to obtain $\rho_{a}$.

g) Next, in the plotted graph, the corresponding probe spacing was obtained.

h) Lastly, the depth of the top layer of soil, $h$ was calculated by using the appropriate probe separation, $a$.

\section{3) Two-layer soil model}

A two-layer soil model was presented by top layer of soil with definite depth whereas the bottom layer of soil with indefinite depth. In this work, two-layer soil model was selected as the soil model used for the grid design. One of the reasons was the measured soil resistivity varied significantly and it was also stated that the development of uniform soil model required almost constant or only slightly changed of soil resistivity according to IEEE Std 80;2013.

Conductors were parts of grounding system that played important role in ensuring the safety of electrical components and any individual at the substation. As important as its role, conductors with proper selection would able to maintain the integrity of a grounding system for long term.

In this work, copper with commercial hard drawn was selected as the grounding conductors for the grid design. Since there were no actual impedances and resistances used, the fault current, $I$ or $3 I_{o}$ was assumed for $20 \mathrm{kA}$. At the same time, the diameter of grounding conductor, $d$ used during the

\section{E. Mathematical Calculation}

\section{1) Two-layer soil model}

The change of resistivity between these two layers was described with reflection factor, $K$. The reflection factor, $K$ can be defined as (1) in the following;

\section{Determine Conductor Size} fieldwork was $20 \mathrm{~mm}$.

$$
K=\frac{p_{2}-p_{1}}{p_{1}+p_{2}}
$$

\section{2) Tolerable step and touch voltages}

The criteria amount of shock from the faults would important to be limit as much as possible before the safety of an individual could be endangered. The limit of these shocks which lead to maximum dangerous voltage was defined as following below;

$$
\begin{aligned}
& E_{\text {step } 50}=\left(1000+6 \times C_{s} \times \rho_{s}\right) \times \frac{0.116}{\sqrt{t_{s}}} \\
& E_{\text {touel }_{s} 50}=\left(1000+1.5 \times C_{g} \times p_{s}\right) \times \frac{0.116}{\sqrt{\mathrm{t}_{s}}}
\end{aligned}
$$

\section{3) Total effective length of ground conductors}

Since grounding rods were used in this grid design, the total effective length of ground conductors, $L_{T}$ was defined as (4);

$$
L_{T}=L_{c}+L_{R}
$$

(4) was then furthered expand into (5);

$$
L_{T}=\left(2 \times n_{\text {side }} \times L_{x}\right)+\left(n_{R} \times L_{y}\right)
$$

\section{4) Grid resistance}

Substation with a proper and good grounding system would provide a low grid resistance, $R_{g}$ to minimize the ground potential rise voltage. The grounding system with such features should have grid resistance of $10 \Omega$ or lower. To calculate the grounding resistance, the formula was defined as (6) in the following;

$$
R_{g}=\rho\left[\frac{1}{L_{T}}+\frac{1}{\sqrt{20 A}}\left(1+\frac{1}{1+\frac{h}{\sqrt{\frac{20}{A}}}}\right)\right]
$$

\section{5) Grid maximum current}

Fault current division factor is the ratio of grid current flowed between the grounding grids to symmetrical fault current that represented by a factor was defined as (7);

$$
S_{f}=\frac{I_{g}}{I_{f f}}
$$

The maximum grid current was defined as (8) as shown in the following;

\section{Published By:}




\section{Design of A Substation Grounding System for Fast Front Overvoltage}

$$
I_{G}=D_{f} \times I_{g}
$$

\section{6) Ground Potential Rise}

The GPR was the product of grid current and grid resistance that defined as (9);

$$
G P R=I_{G} \times R_{g}
$$

\section{7) Mesh and step voltages}

The mesh voltage was defined as (10) in the following;

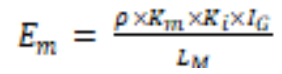

The step voltage was defined as (11) in the following;

$$
E_{s}=\frac{\rho \times K_{s} \times K_{i} \times I_{L}}{L_{w}}
$$

\section{8) Geometrical factors of step and mesh voltages}

For geometrical factor, $K_{m}$, the calculation for each $K_{i i}$ and $K_{h}$ are shown in the following;

$$
K_{\text {ii }}=1
$$

$$
K_{h_{h}}=\sqrt{1+\frac{h_{h}}{h_{g}}} \text { where } \mathrm{h}_{\mathrm{o}}=1
$$

$$
\begin{aligned}
& K_{m}=\frac{1}{2 \pi} \times\left[\operatorname { l n } \left[\frac{D^{2}}{16 \times h \times d}+\frac{(D+2 \times h)^{2}}{8 \times D \times d}\right.\right. \\
& \left.\left.-\frac{\hbar}{4 \times d}\right]+\frac{K_{\hat{h}}}{K_{h}} \times \operatorname{In}\left[\frac{\mathrm{g}}{\pi(2 \times n-1)}\right]\right]
\end{aligned}
$$

The geometrical factor, $K_{s}$ was defined in (15) as followed;

$$
K_{s}=\frac{1}{\pi}\left[\frac{1}{2 \times h}+\frac{1}{D+h}+\frac{1}{D}\left(1-0.5^{n-2}\right)\right]
$$

\section{9) Irregularity factor}

The irregularity factor, $K_{i}$ was defined in (16) as followed;

$$
K_{\mathrm{i}}=0.644+0.148 \times n
$$

10) Geometrical factor of grid

$$
n=n_{a} \times n_{b} \times n_{c} \times n_{d}
$$

where

$$
n_{a}=\frac{2 \times L_{c}}{L_{p}}
$$

$$
\begin{aligned}
& n_{b}=1 \text { for square grids } \\
& n_{c}=1 \text { for square and rectangular grids } \\
& n_{d}=1 \text { for square, rectangular and L-shaped grids }
\end{aligned}
$$

\section{RESULTS AND DISCUSSION}

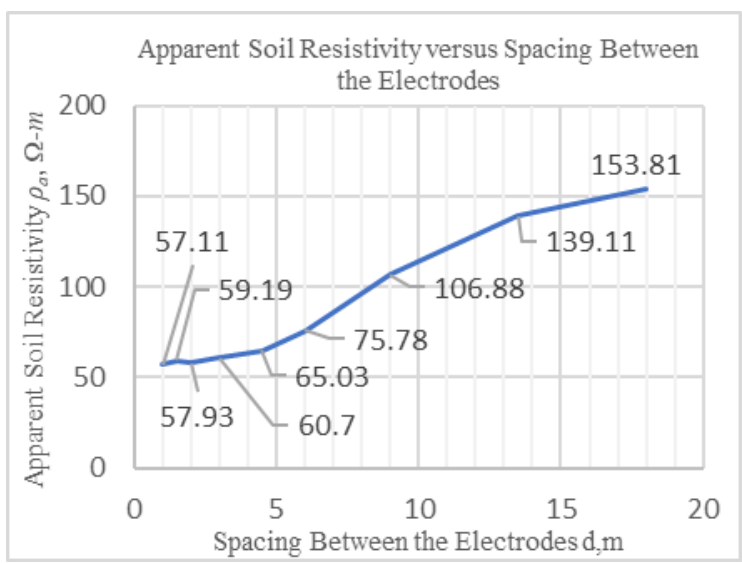

Fig. 4. The graph of apparent soil resistivity versus spacing between electrodes.

In Fig. 4, the soil data obtained from fieldwork is displayed in the form of graph where the y-axis represents the soil resistivity, $\Omega-m$ and x-axis represents the probe spacing, $a$. It is an upward trend as soil resistivity increases when the probe spacing increases showing that the top layer of soil resistivity is lower than the bottom layer of soil resistivity.

From this work, the surface material of washed granite is used as thin layer on the designed grid. It is to minimise the danger faced by the personnel or power equipment within the vicinity when there is fault. The reflection factor, $K$ is -0.9763 and it indicates that the current density is uniformly spread across the grounding grid due to tendency of grid current flows downward into lower resistivity of soil layer instead of higher resistivity one.

I. THE COMPARISON BETWEEN TOLERABLE VOLTAGES WITH STEP AND MESH VOLTAGES.

\begin{tabular}{|l|c|}
\hline Parameter & Value \\
\hline Tolerable step voltage $\left(E_{\text {step }, 50}\right)$ & 3298.52 \\
\hline Step voltage $\left(E_{s}\right)$ & $695.29 \mathrm{~V}$ \\
\hline Tolerable touch voltage $\left(E_{\text {touch, } 50}\right)$ & $947.67 \mathrm{~V}$ \\
\hline Mesh voltage $\left(E_{m}\right)$ & $814.11 \mathrm{~V}$ \\
\hline
\end{tabular}

In Table I, the mesh voltage is $814.11 \mathrm{~V}$ which is lower than $947.67 \mathrm{~V}$ for the tolerable touch voltage, $E_{\text {touch,50. The }}$ step voltage is $695.29 \mathrm{~V}$ which is also lower than $3298.52 \mathrm{~V}$ for the tolerable step voltage, $E_{\text {step,50. High step voltage }}$ means the difference in voltage between two points from grounding point is large and a person could be at risk of injury easily. From the result, the tolerable step voltage is able to provide protection against risk of injury from the magnitude of step voltage. Next, mesh voltage is the difference in voltage between the metallic object connected to the grid and the soil potential within the grid. From the 
result, the grid is considered as safe.

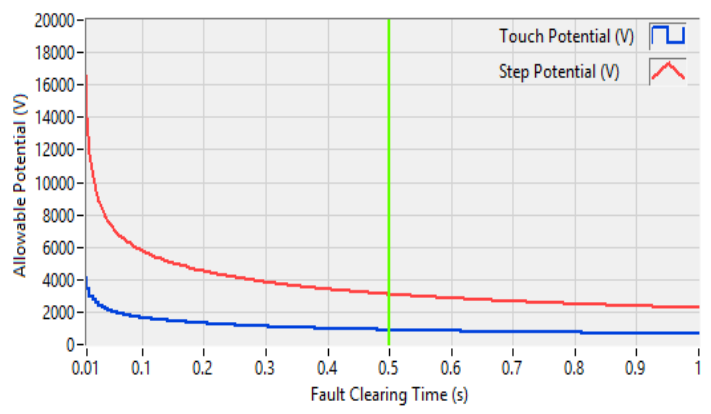

Fig. 5. The simulation result for tolerable step and touch voltages.

Fig. 5 shows the simulation of tolerable voltages obtained in SafeGrid Earthing software. The computed values are then compared with the calculated values to verify data for grid design. Table II shows the comparison between calculated values and computed values as well the percentage of error. For tolerable step voltage, calculated value is $3298.52 \mathrm{~V}$ and its computed value is $3080.10 \mathrm{~V}$ with percentage of error by $7.09 \%$. For tolerable touch voltage, calculated value is $947.67 \mathrm{~V}$ and its computed value is $885.90 \mathrm{~V}$ with percentage of error by $6.97 \%$. Table III shows the overview for all parameters and materials used in this grid design.

\section{THE COMPARISON BETWEEN CALCULATED AND} COMPUTE VALUES.

\begin{tabular}{|l|l|l|l|}
\hline Parameter & $\begin{array}{l}\text { Calculated } \\
\text { Voltage, V }\end{array}$ & $\begin{array}{l}\text { Computed } \\
\text { Voltage, V }\end{array}$ & $\begin{array}{l}\text { Percentage } \\
\text { of Error, \% }\end{array}$ \\
\hline Tolerable Step Voltage & 3298.52 & 3080.10 & 7.09 \\
\hline Tolerable Touch Voltage & 947.67 & 885.90 & 6.97 \\
\hline
\end{tabular}

\section{THE OVERALL SUMMARY OF GRID DESIGN.}

\begin{tabular}{|c|c|c|c|}
\hline No. & Parameter Description & Value & Unit \\
\hline 1 & Surface material & $\begin{array}{l}\text { Washed } \\
\text { granite }\end{array}$ & \\
\hline 2 & Surface material resistivity $\left(\rho_{s}\right)$ & 5000 & $\Omega-\mathrm{m}$ \\
\hline 3 & Surface layer thickness $\left(h_{s}\right)$ & 0.08 & $\mathrm{~m}$ \\
\hline 4 & Soil resistivity $(\rho)$ & 60 & $\Omega-\mathrm{m}$ \\
\hline 5 & Symmetrical fault current $\left(3 I_{o}\right)$ & $20 \times 10^{3}$ & A \\
\hline 6 & Type of grounding conductor & Copper & \\
\hline 7 & Diameter of grounding conductor $(d)$ & 0.020 & $\mathrm{~m}$ \\
\hline 8 & Duration of fault current $\left(t_{c}\right)$ & 0.50 & $\mathrm{~s}$ \\
\hline 9 & Reflection factor, $K$ & -0.9763 & \\
\hline 10 & Surface layer derating factor $\left(C_{s}\right)$ & 0.6369 & \\
\hline 11 & Tolerable step voltage $\left(E_{\text {step, } 50}\right)$ & 3298.52 & $\mathrm{~V}$ \\
\hline 12 & Tolerable touch voltage $\left(E_{\text {touch }, 50}\right)$ & 947.67 & $\mathrm{~V}$ \\
\hline 13 & Spacing between equally conductor $(D)$ & 4 & $\mathrm{~m}$ \\
\hline 14 & $\begin{array}{l}\text { Total effective length of ground conductor } \\
\left(L_{T}\right)\end{array}$ & 940 & $\mathrm{~m}$ \\
\hline 15 & $\begin{array}{l}\text { Geometry factor composed of } n_{a}, n_{b}, n_{c}, n_{d} \\
(n)\end{array}$ & 11 & \\
\hline 16 & Grid resistance $\left(R_{g}\right)$ & 0.70 & $\Omega$ \\
\hline 17 & Fault current division factor $\left(S_{f}\right)$ & 0.60 & \\
\hline 18 & Symmetrical grid current $\left(I_{g}\right)$ & $12 \times 10^{3}$ & $\mathrm{~A}$ \\
\hline 19 & $\begin{array}{l}\text { Decrement factor for the entire duration of } \\
\text { fault }\left(D_{f}\right)\end{array}$ & 0.60 & \\
\hline 20 & Maximum grid current $\left(I_{G}\right)$ & $12 \times 10^{3}$ & $\mathrm{~A}$ \\
\hline 21 & Ground potential rise (GPR) & $8.4 \times 10^{3}$ & $\mathrm{~V}$ \\
\hline 22 & Geometrical factor for mesh voltage $\left(K_{m}\right)$ & 0.4852 & \\
\hline 23 & Irregularity factor for grid geometry $\left(K_{i}\right)$ & 2.272 & \\
\hline 24 & Effective buried length for mesh voltage $\left(L_{m}\right)$ & 974.94 & $\mathrm{~m}$ \\
\hline 25 & Mesh voltage $\left(E_{m}\right)$ & 814.11 & $\mathrm{~V}$ \\
\hline 26 & Geometrical factor for step voltage $\left(K_{s}\right)$ & 0.3022 & \\
\hline 22 & Effective buried length for step voltage $\left(L_{s}\right)$ & 711 & \\
\hline 23 & Step voltage $\left(E_{S}\right)$ & 695.29 & $\mathrm{~V}$ \\
\hline
\end{tabular}

\section{ACKNOWLEDGMENT}

The author would like to express the gratitude and thank you to his supervisor and moderator for their full support and guidance in this work. Special thanks to Faculty of Engineering Multimedia University (Cyberjaya campus) for allowing the author to perform this work as part of final year project.

\section{REFERENCES}

1. U. M. Angst, and B. Elsener, "On the Applicability of the Wenner Method for Resistivity Measurements of Concrete," ACI Materials Journal, 111(6), 2014.

2. R. D. Southey, M. Siahrang, S. Fortin, and F. P. Dawalibi, "Using fall-of-potential measurements to improve deep soil resistivity estimates," IEEE Transactions on Industry Applications, 51(6), pp. 5023-5029, 2015.

3. K.M. Prakash, J.S. Chirag, and B.A. Riya, "Design of Substation Grounding In A Two Layer Earth Structure (A Case Study Of 132kV Substation)," in International Journal of Advanced Technology in Engineering and Science. pp. 11-21, 2017.

4. G. Gilbert, Y. L. Chow, D. E. Bouchard, and M. M. A. Salama, "Soil model determination using asymptotic approximations to Sunde's curves," inTransmission and Distribution Conference and Exposition, 2010 IEEE PES. pp.1-7, April. 2010.

5. A.L. Vikram, Vaghamshi, and K.A, Sonagra, "Substation Grounding Grid Design Case Study Using Matlab," in International Journal for Scientific Research and Development. pp. 748-751, 2014.

6. P. Kapijan, S. Potivejkul, and P. Yutthagowith, "Grounding system design of substation using Matlab program," in Electrical Engineering Congress (iEECON) International pp. 1-4, March. 2017.

7. K. A. Vyas, and J. G. Jamnani, "Optimal design and development of software for design of substation grounding system," in Engineering (NUiCONE), Nirma University International Conference. pp. 1-7, December. 2011.

8. IEEE Guide for Safety in AC Substation Grounding, IEEE Std.80: 2013.

\section{AUTHORS PROFILE}

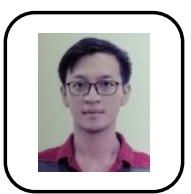

Kevin Lee Yuan Jet is previously a Bachelor of Degree in Electrical holder in Multimedia University, Cyberjaya Campus. During his final year project, he engaged with Dr $\mathrm{Ngu}$ and Dr Normiza in consultation and conducting experimental tests related to the properties of soil at Multimedia University. He completed his Degree studies in 2018 and currently working as Mill Engineer in IJM Plantation.

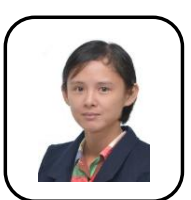

Ngu Eng Eng is currently a Senior Lecturer at Faculty of Engineering, Multimedia University, Cyberjaya Campus. She obtained her Doctor of Philosophy $(\mathrm{PhD})$ in Electrica Engineering (Power System) in 2012. During these years, Dr. Ngu has published many journals and proceedings related with transmission lines and fault location studies. In Multimedia University, she is an Assistant Programme Coordinator for Electrical Program, member of Industrial Linkage Committee and Internal Maintenance Audit for Faculty.

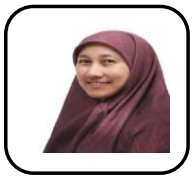

Normiza Binti Mohamad Nor is currently an Associate Professor of Electrical Engineering at Faculty of Engineering, Multimedia University, Cyberjaya Campus. She completed her Doctor of Philosophy $(\mathrm{PhD})$ in Electrical Engineering, University of Wales, College of Cardiff, United Kingdom in 2002. Dr. Normiza has published many journals and proceedings for the past 20 years. She also involved with consultancy works and delivered seminars in her area of expertise such as high voltage engineering, grounding system and lightning protection system. 